\title{
Editorial: Topical Volume on Dust Devils
}

\author{
Dennis Reiss ${ }^{1}$ Ralph D. Lorenz ${ }^{2}$ Matthew Balme ${ }^{3}$. \\ Lynn D. Neakrase ${ }^{4}$. Angelo P. Rossi ${ }^{5}$. Aymeric Spiga ${ }^{6}$. \\ John Zarnecki ${ }^{7}$
}

Published online: 16 November 2016

(C) Springer Science+Business Media Dordrecht 2016

Dust devils have captivated humankind since antiquity, and yet also attained importance in planetary exploration. The close investigation of the planet Mars by an armada of spacecraft in the last couple of decades has stimulated extensive research on these desert whirlwinds,

\author{
D. Reiss \\ dennis.reiss@uni-muenster.de \\ R.D. Lorenz \\ ralph.lorenz@jhuapl.edu \\ M. Balme \\ matt.balme@open.ac.uk \\ L.D. Neakrase \\ lneakras@nmsu.edu \\ A.P. Rossi \\ an.rossi@jacobs-university.de
}

\section{A. Spiga}

aymeric.spiga@upmc.fr

J. Zarnecki

zarnecki@issibern.ch

1 Institut für Planetologie, Westfälische Wilhelms-Universität, Wilhelm-Klemm-Str. 10, 48149 Münster, Germany

2 Applied Physics Laboratory, Johns Hopkins University, 11100 Johns Hopkins Road, Laurel, MD 20723, USA

3 Dept. of Physical Sciences, Open University, Walton Hall, Milton Keynes MK7 6AA, UK

4 Department of Astronomy, New Mexico State University, Las Cruces, NM, USA

5 Department of Physics and Earth Sciences, Jacobs University Bremen, Campus Ring 1, 28759, Bremen, Germany

6 Laboratoire de Météorologie Dynamique, Université Pierre et Marie Curie, Paris, France

7 International Space Science Institute, 3012 Bern, Switzerland 
supported by improvements in field instrumentation on Earth, laboratory experiments and powerful numerical simulations. This convergence of capability and interest stimulated the idea to produce a state-of-the art review volume on Dust Devils. As is known in the space sciences communities, the International Space Science Institute offers a platform for such a purpose, the ISSI workshops. 'Dust Devils on Mars and Earth', was conceived by Dennis Reiss, Ralph Lorenz, Matt Balme, Lynn D. Neakrase, Angelo P. Rossi, Aymeric Spiga and John Zarnecki, held under the auspices of the International Space Science Institute in Bern, Switzerland, during the week of February 16-20, 2015. The workshop drew not only planetary scientists, for whom terrestrial dust devils are a convenient proxy for their Martian cousins, but also scientists studying the impact of dust on the terrestrial climate. The outcome is presented in this Topical volume of Space Science Reviews:

Review article 1 by Lorenz et al. surveys the historical development of dust devil studies, noting a vivid description of one in Sophocles' writings 25 centuries ago, the beginnings of scientific investigation in the 19th century and the systematic improvements in field research until the early 1970s. Mars exploration seems then to have been an additional stimulus to dust devil studies, particularly following the Pathfinder and Mars Global Surveyor missions of 1997. This paper also reviews the occasionally damaging nature of dust devils, responsible for a few deaths per decade, and the role of dust devils in culture.

Field observations, by researchers on Earth and robots on Mars, are reviewed in Paper 2 (Murphy et al.). Remarkably, it is only in recent years that high-time-resolution field meteorological data for dust devil sites on Earth have become widely available; generally field data from Mars have been better! Terrestrial field measurements have quantified an extensive range of vortex parameters such as rotational, vertical and translational speeds, dust loads, and pressure deficits. Dust devil parameters obtained on Earth are more temporally limited compared to the long-term operating robotic missions on Mars. The latter have enabled the quantification of daily and seasonal dust devil occurrence on Mars. Recent developments measuring dust devils with electric field, infrasound, acoustic and seismic sensors on Earth and its application in future missions on Mars are discussed.

Dust devils were first seen on Mars from above, by the Viking orbiter cameras (the Viking landers' slow-scan facsimile cameras were not suitable for observing moving objects) and these and other orbital observations since are reviewed in Paper 3 (Fenton et al.). Orbital imaging at Mars has progressed dramatically in coverage and resolution, and useful dust devil statistics have emerged, noting a number of regions of particularly enhanced activity. Recent work has shown that dust devil height and spacing can be related to large-scale ambient properties, such as the thickness of the boundary layer, and dust devil tracking provides much-needed insight into Mars' near-surface winds. Dust raising by other processes (notably dust storms) is important on both Mars and Earth, and orbital observations of dust raising generally on both worlds is reviewed in this article.

Some of the most numerous and prominent ephemeral surface features on Mars are dark or bright surficial tracks left by the passages of dust devils, commonly named 'dust devil tracks'. In the last decades dust devil tracks have also been identified on Earth in highresolution satellite imagery and have been subsequently studied in situ. Their different morphological types, formation mechanisms, and spatial and temporal distribution on Mars and Earth are reviewed in Paper 4 (Reiss et al.). Dust devil tracks as proxies for dust devil activity, ambient wind conditions, dust devil lifetimes, and solar panel clearing events are discussed. Their impact on the climate on Mars due to large-scale surface albedo changes and the current state-of-the-art in automatic detection of dust devil tracks is more generally discussed.

The conditions under which dust devils form are reviewed in Paper 5 (Rafkin et al.). The association of dust devils with strong surface heating is well-established, but the anecdotal 
observation of an 'optimum' wind speed range for dust devil occurrence was not so obvious. Datasets and numerical simulations supporting this effect are reviewed, and the generation of vorticity more generally is discussed.

The nature of dust devils as discrete, self-sustaining convective vortices has long attracted the attention of workers in fundamental fluid mechanics. Various idealizations of a vortex equating the centripetal acceleration of air parcels to that due to a pressure gradient, with various analytic descriptions of wind and pressure fields since the original 'Rankine vortex' model of the 19th century are reviewed in Paper 6 (Kurgansky et al.). A thermodynamical theory that explains how the vortices are maintained and provides an order-of-magnitude estimate of their principal parameters is also overviewed, as well as discussed in this paper are the effects of vortex translational motion, of surface roughness and of lofted dust on the steady-state structure of dust devils.

Among the most powerful tools in contemporary dust devil studies is the computer, through the framework of (most aptly-named in this case) Large-Eddy Simulation (LES) described in review article 7 (Spiga et al.). These simulations describe the three-dimensional fields of pressure, velocity etc. in the planetary boundary layer, and explicitly resolve the vortex structures that emerge spontaneously therein. This approach allows the visualization of flow structure that is generally impossible to see in the field, and brings a perspective of dust devils not so much as discrete entities, but as part of a convecting flow system. Simulations, which have been performed for both Earth and Mars conditions, allow the tracking of the lifetime of individual structures, and their growth and sometimes merging. These numerical experiments, which nonetheless often involve many idealizations, allow systematic evaluation of the influence of environmental parameters such as ambient windspeed, on dust devil populations.

Review article 8 (Lorenz and Jackson) assesses the challenge of systematically evaluating dust devil populations, given their sporadic nature and extremely skewed size and intensity distributions: like earthquakes, there are many more small ones than large ones. Statistical techniques for comparing and portraying the level of activity, as determined by a variety of observational techniques, are reviewed. It is concluded that the widely-held assumption that 'Martian dust devils are larger than those on Earth' is not strongly supported by data, given the very different techniques used to survey their populations (different scales of observation sample different parts of the size distribution), but the best comparisons do indeed suggest a factor of about 3 in median size, and it is certainly true that the largest dust devils documented are on Mars. The paper also notes the nonstationary statistics of dust devils, that they occur in 'bursts' when conditions are favorable, and may do so in a pseudoperiodic manner.

The electrical properties of dust devils have been studied since the 1860s, and charge generation in dust is reviewed in Paper 9 (Harrison et al.). Planetary atmospheric electricity research has been stimulated by instrumentation development around the launch of an electric field sensor to Mars on the Schiaparelli lander in 2016, and field experiments on dust charging associated with that investigation. In this paper the causes of atmospheric dust electrification in general are discussed and future instrumentation for electric field measurements on Mars are reviewed.

The particle-lifting process is reviewed at a microscopic level in Paper 10 (Neakrase et al.): a fundamental challenge for both Earth and Mars, but especially the latter, is that modeled flow speeds to launch dust are improbably high due to the strong cohesion between very small dust grains. Various physical processes to circumvent this apparent 'lifting threshold speed' barrier are reviewed, illuminated especially by laboratory experiments. Among possible contributors are sand-blasting (using easier-moved large grains to dislodge smaller 
ones), the suction or 'Delta-P' effect, wherein the vortex pressure drop causes the surface to exhaust pore gas upwards, the break-up of large dust aggregates, the role of electrostatic force, and even exotic light-driven processes. It is concluded that all these effects may play a role, and the dominant ones may vary from location to location.

The problem of how to scale up dust lifting observed in individual devils, to the dust devil input to the overall aerosol content of the atmosphere, is reviewed in Paper 11 (Klose et al.). Field observation and numerical simulation results can inform the parameterizations used in global models to estimate dust fluxes. Since global models cannot hope to resolve individual devils, population statistics and functional relationships between dust fluxes and ambient parameters must be devised. On Mars the role of dust devils is considered to be important, contributing tens of per cent to the total, whereas on Earth it is small at the global scale.

This synthesis of the state-of-the-art in dust devil studies from two planets, Earth and Mars, serves as a basis for a better understanding of dust devil processes and quantifying their global dust entrainment contribution into the atmospheres of both planets. Unresolved questions and future directions in dust devil research are outlined in this Topical volume of Space Science Reviews to stimulate further research and future robotic missions on Mars.

Acknowledgements The Convenors of the workshop "Dust Devils on Earth and Mars" would like to express their sincere appreciation to ISSI, in particularly to Maurizio Falanga, Jennifer Fankhauser-Zaugg, Saliba Saliba, Andrea Fischer, and Silvia Wenger for their very kind and excellent organization of this workshop as well as their technical and editorial help. The Editors would also like to thank the authors who contributed to this issue and all reviewers who contributed their time and expertise in reviewing the manuscripts. 